\title{
Statistical Bubble Localization with Random Interactions
}

\author{
Xiaopeng Li, Dong-Ling Deng, Yang-Le Wu, and S. Das Sarma \\ Condensed Matter Theory Center and Joint Quantum Institute, \\ Department of Physics, University of Maryland, College Park, MD 20742-4111, USA
}

\begin{abstract}
We study one-dimensional spinless fermions with random interactions, but without any on-site disorder. We find that random interactions generically stabilize a many-body localized phase, in spite of the completely extended single-particle degrees of freedom. In the large randomness limit, we construct "bubble-neck" eigenstates having a universal area-law entanglement entropy on average, with the number of volume-law states being exponentially suppressed. We argue that this statistical localization is beyond the phenomenological local-integrals-of-motion description of many-body localization. With exact diagonalization, we confirm the robustness of the many-body localized phase at finite randomness by investigating eigenstate properties such as level statistics, entanglement/participation entropies, and nonergodic quantum dynamics. At weak random interactions, the system develops a thermalization transition when the single-particle hopping becomes dominant.
\end{abstract}

Disorder in isolated quantum systems leads to fascinating phenomena such as Anderson localization [1]. Noninteracting particles in the Anderson localized phase form a perfect insulator with vanishing DC conductivity even at infinite temperature. The lack of thermal transport in an Anderson localized system prohibits thermalization, making it intrinsically nonergodic and far out of equilibrium. The stability of localization and non-ergodicity against interactions, however, remained controversial until the recent study of many-body localization (MBL) [24]. Following the perturbative analysis in Ref. [2], the robustness of localization against interactions has now been established through exact numerical calculations [515] and a mathematical proof under certain reasonable assumptions [16]. Experimentally, the dynamical nonergodic aspects of the MBL phase have been examined with cold atoms in optical lattices [17-20] and trapped ions [21]. Although currently an active area of research, the general consensus is that a noninteracting quantum system with sufficiently strong single-particle (i.e. onsite) disorder remains many-body-localized in the presence of finite interparticle interactions.

While the existence of MBL is accepted for interacting disordered fermions, the role of interaction remains somewhat tangential. In the numerical studies of models with on-site disorder, MBL is only found in the regime dominated by single-particle disorder potentials where the noninteracting system is necessarily strongly localized [5-11]. Mathematically, despite the proof of existence of MBL [16], a lower bound for the required disorder strength has not been established. In the "localintegrals-of-motion" (LIM) description [22-25], the conserved charges strongly resemble their non-interacting counterparts in the deep MBL regime. It is difficult to single out the effect of interaction for MBL in models with single-particle disorder, where interaction and single-particle terms are always intertwined. This issue is particularly worrisome when one looks for "smokinggun" experimental signatures to distinguish MBL from Anderson localization, and the possibility that all experimentally observed MBL phenomena are essentially (slightly perturbed) single-particle Anderson localization cannot be definitively ruled out. It is thus desirable to study a simpler system where the localization is driven purely by many-body effects, and the interacting MBL phase is not adiabatically connected to a single-particle Anderson localized phase.
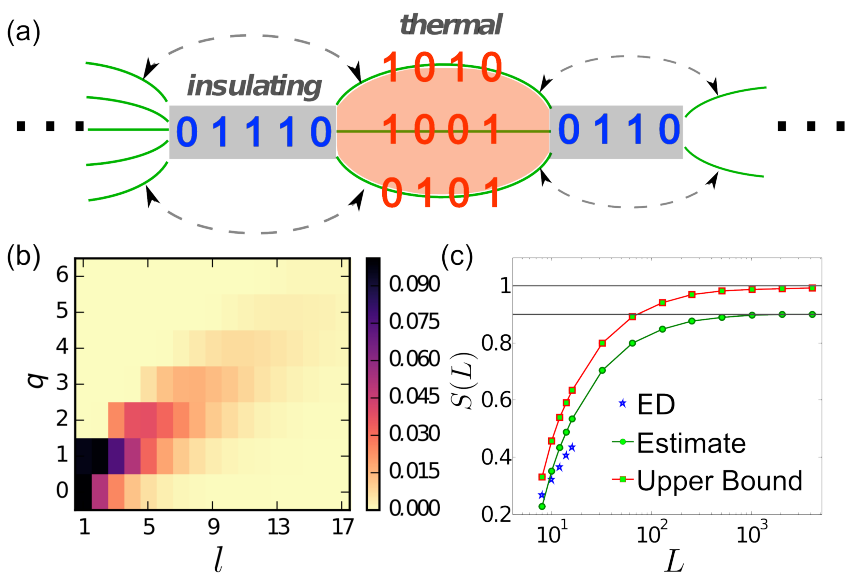

(c)

Figure 1. Eigenstates in the infinite random interaction limit. (a) illustrates the "bubble-neck" eigenstates. Clusters with more than one fermion on adjacent sites form insulating blocks (IB). Others with isolated fermions form thermal bubbles (TB). Quantum superpositions are allowed (forbidden) in the TB (IB). Cross-block tunnelings (dashed arrows) are negligible in this limit. (b) shows the histogram of the size $(l)$ and the particle number $(q)$ of TBs. (c) shows the bipartite entanglement entropy. The symbols ' $t$ ' correspond to exact diagonalization results with $W / t=10^{3}$. The solid lines correspond to the entanglement upper bound and the Page-value estimate from random sampling, which respectively saturate to $S_{\mathrm{ub}} \approx 1$ and $S_{\mathrm{est}} \approx 0.9$ in the $L \rightarrow \infty$ limit.

In this paper, we consider the precise opposite limit and study MBL in a random-interaction model, whose non-interacting limit is completely extended. In the strong randomness limit, we formulate a "bubble-neck" construction (see Fig. 1) for the MBL eigenstates in this system. Such bubble-neck eigenstates could have volumelaw entanglement. Our construction hence goes beyond the scope of the LIM description and describes a novel 
type of MBL with no non-interacting analogue whatsoever (i.e. the corresponding noninteracting system is in a trivial extended phase). Further, we show that the average entanglement entropy over all such eigenstates still obeys an area law, and we provide a generic entropy upper bound, independent of the specific model realization of thermal bubbles. With exact numeric calculations, we confirm the robustness of the MBL phase at finite random interactions. For weak disorder, the system develops a thermalization transition when the single-particle tunneling effects become dominant overwhelming random interaction effects. We stress that our proposed statistical bubble MBL phase is driven solely by the interaction, without any influence from single-particle on-site disorder. While aspects of MBL in the presence of extended single-particle orbitals have been discussed in other systems [26-31], our work shows that clean interacting spinless fermions have novel generic features distinct from previous studies, establishing that MBL in clean random interacting fermion systems is a generic phenomenon completely distinct from the MBL physics in disordered interacting systems which are adiabatically connected to Anderson localized systems as the interaction is turned off.

Model.-We study one-dimensional (1D) spinless fermions with random nearest neighbor interactions,

$$
H=-t \sum_{j=1}^{L}\left[c_{j}^{\dagger} c_{j+1}+H . c .\right]+\sum_{j} V_{j} n_{j} n_{j+1},
$$

where $c_{j}$ is a fermonic annihilation operator, $n_{j}=c_{j}^{\dagger} c_{j}$, $L$ is the number of lattice sites, and the tunneling $t$ is the energy unit throughout this paper. We consider a uniform distribution for the random interactions $V_{j} \in[-W, W]$ and focus on half-filling. In this model, the disorder effects arise purely from interactions, with the non-interacting degrees of freedom being completely delocalized.

Analysis of the infinite randomness limit. - Let us first consider the strong randomness limit $W \rightarrow \infty$. If the tunneling $t$ is strictly zero, the eigenstates of the system are trivial product states albeit with huge degeneracies. Turning on an infinitesimal tunneling breaks the degeneracy and gives a bubble-neck structure to the eigenstates to be described below.

With infinitesimal tunneling (to the leading order in $t / W)$, a cluster with more than one particles on adjacent sites (Fig. 1) is localized (i.e., does not tunnel) due to random two-body interactions, and such clusters form insulating blocks. Other clusters with isolated fermions are extended, forming thermal bubbles. Fermions in the thermal bubbles can tunnel almost freely, except that the configurations with two fermions coming to adjacent sites are forbidden. A thermal bubble with $l$ lattice sites and $q$ fermions has a Hilbert space dimension $D_{\text {therm }}(l, q)=\left(\begin{array}{c}l+1-q \\ q\end{array}\right)$. Fermion tunneling in a thermal bubble makes a finite many-body energy splitting of the order of $t / D_{\text {therm, }}$, which prohibits couplings of different
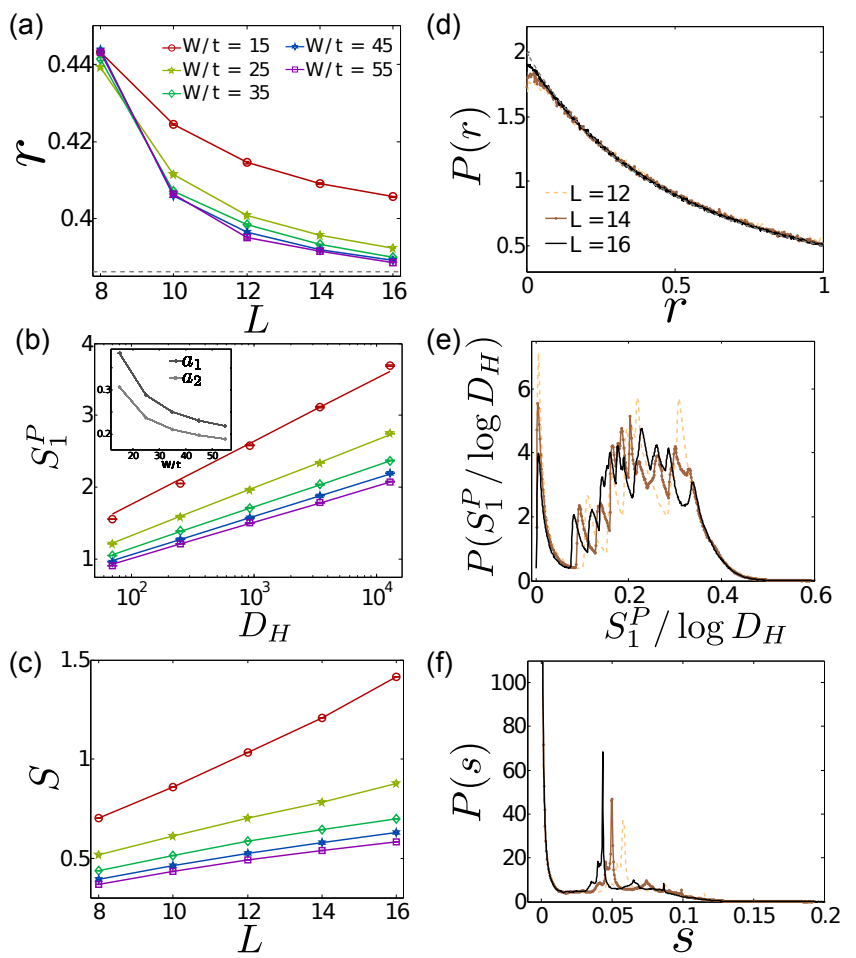

Figure 2. The MBL phase of the random interaction model (Eq. (1)). (a), (b), and (c) share the same legend and show the system-size dependence of $r, S_{1}^{P}$, and $S$, respectively. Their probability distributions across disorder samples and different eigenstates in the deep localized phase $(W / t=55)$ are shown in (d), (e), and (f). The dashed line in (a) marks the Poisson value $r_{P}=2 \log 2-1$. In $(\mathrm{d})$, the numeric data for $P(r)$ with $W / t=55$ for different $L$ collapse to $P_{0}(r)=2 /(1+r)^{2}$ (grey dashed line) with the deviation barely noticeable in this plot. The inset in (b) shows the participation entropy coefficients (see main text).

thermal bubbles across insulating blocks (to leading order in $t / W)$. The resulting bubble-neck eigenstates are illustrated in Fig. 1. In the infinite randomness limit, only the thermal bubbles contribute to the entanglement entropy. With random state sampling [32], we find that the probability distribution of the thermal-bubble-size $P(l, q)$ decays exponentially for large $l$ (Fig. 1b). The entanglement entropy of the eigenstates in the large randomness limit is thus bounded, i.e., obeying an area-law scaling, which implies that the system is many-body localized (see Fig. 1c for the explicit entanglement scaling). We find that the area-law entanglement entropy of such bubble-neck eigenstates has a generic upper bound with the Page-value estimate [33] in the thermodynamic limit,

$$
\begin{aligned}
& S_{\mathrm{ub}}(L \rightarrow \infty) \approx 1, \\
& S_{\mathrm{est}}(L \rightarrow \infty) \approx 0.9,
\end{aligned}
$$

independent of the specific model of thermal bubbles. Here the Page-value is the entanglement entropy averaged over random pure states [33], and it provides an estimate for the entanglement in thermal states [34]. The 
Page-value estimate agrees with our numeric exact diagonalization results for small systems (Fig. 1c). We emphasize that the MBL eigenstates in the infinite interaction disorder limit are generic, independent of the specific disorder realizations. The bubble-neck MBL picture with generic statistical entanglement properties does not depend on the specific model of the dynamics in the thermal bubble.

We stress that our MBL phase goes beyond the LIM description. In the LIM picture [22-25], all eigenstates for a fixed disorder configuration are short-range entangled with their entanglement entropy determined by certain localization length. In sharp contrast, the generic bubble-neck eigenstates (Fig. 1a) could be long-range (volume-law) entangled although the number of such states is statistically suppressed by the exponentially decaying probability of long bubbles (Fig. 1(b)). We thus conclude that our proposed random interaction driven MBL phase is sharply distinct from the on-site disorder driven MBL.

It is worth noting that the thermal bubble of the particular model in Eq. (1) is actually integrable through an inflated-fermion mapping approach [32]. However we stress that the physics presented here does not rely on the choice of this particular model. We check this by replacing the single-particle Hamiltonian with the AubryAndré model where the thermal bubble is no longer integrable, finding quantitatively similar results [32].

The MBL phase at finite randomness. - With finite random interaction, the "forbidden" cross-block couplings (Fig. 1a) come into play and our bubble-neck picture no longer strictly applies. We study such effects using exact diagonalization. We have investigated different diagnostics, the bipartite entanglement entropy $(S)$, the level statistics gap ratio $(r)$, and the wavefunction participation entropy $\left(S_{m}^{p}\right)$, which are widely used in the literature to characterize MBL. The entanglement entropy $S$ signifies localization in real space. The gap ratio that characterizes the level statistics is defined to be $r \equiv \min \left(\delta_{n}, \delta_{n+1}\right) / \max \left(\delta_{n}, \delta_{n+1}\right)$ [5], with $\delta_{n}$ the energy spacing between close-by eigenstates. The participation entropy [35-38] is introduced to quantify the localization property in the many-body Hilbert space, $S_{m}^{P}=\frac{1}{1-m} \sum_{\{n\}}\left|\Psi_{\{n\}}\right|^{2 m}$, with $S_{1}^{P}=$ $-\sum_{\{n\}}\left|\Psi_{\{n\}}\right|^{2} \log \left|\Psi_{\{n\}}\right|^{2}$, where $\Psi_{\{n\}}$ is the many-body wave function. We average over 1000 (10000) disorder realizations for systems with size $L \geq 12(L<12)$. Within each disorder realization, we average over all eigenstates with an equal weight, corresponding to an "infinite temperature" ensemble.

In Fig. 2, we provide the system-size dependence and the probability distributions of different quantities. Fig. 2(a) shows the average gap ratio with varying random interaction strength $W / t$. This quantity approaches the GOE (Gaussian Orthogonal Ensemble) value $r_{G} \approx 0.53$ in the thermal phase and the Poisson value $r_{P}=2 \log 2-1$ in the nonergodic MBL phase. At strong random interaction $(W / t \in[25,55]$ shown in
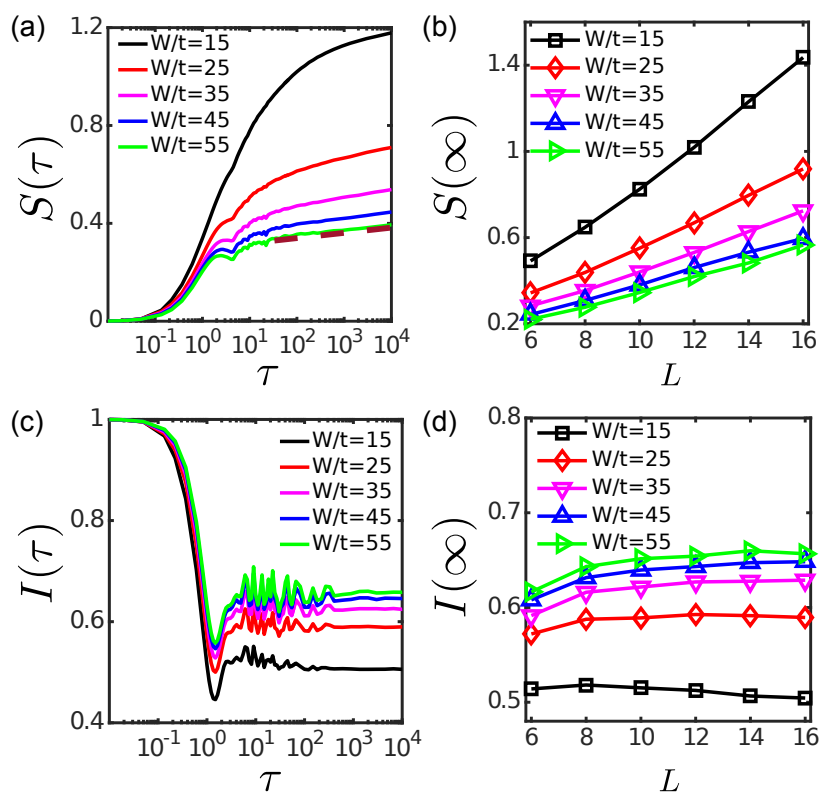

Figure 3. Dynamical properties of the localized phase. Here we begin with random product states and then compute their time-evolution with $H$ defined in Eq. (1). (a) Entanglement growth with varying $W / t$. The dashed brown line is a logarithmic fit to the data. (b) Scaling of the saturated value of entanglement $S(\infty)$. Similar to the case of random on-site disorder driven MBL [7], for strong random interaction with $W>25$, the entanglement entropy exhibits an unbounded logarithmic growth in the thermodynamic limit and the its saturation value obeys a volume-law for finite $L$. This lends strong support of a random interaction driven MBL phase. (c)Dynamical evolution of density imbalance. It saturates to a finite value and thus does not relax at long-time, another signature of MBL and ergodicity breaking. (d) Density imbalance at long time limit as a function of $L$ for different $W / t$.

the figure) $r$ monotonically decreases as we increase the system size, and systematically approaches the universal Poisson value $r_{P}$ in the thermodynamic limit (Fig. 2(a)). Moreover, the probability distribution of the gap ratio for different eigenstates and disorder samples collapses to the function of $P_{0}(r)=2 /(1+r)^{2}$ (Fig. 2(d)), which corresponds to the precise Poisson level statistics. We attribute the small deviation from $P_{0}(r)$ to finite-size effects as it systematically shrinks on increasing $L$.

Fig. 2(b) shows the rank-1 participation entropy $S_{1}^{P}$. In the thermal phase with its wave function completely delocalized in the Hilbert space, $S_{1}^{P}$ will approach $\log D_{H}$ ( $D_{H}$ is the Hilbert space dimension) in the thermodynamic limit, whereas in the localized phase $S_{1}^{P} / \log D_{H}<$ 1 meaning the wave function does not spread over the entire Hilbert space. In our numerics, we find that $S_{1}^{P}$ is proportional to $\log D_{H}, S_{1}^{P}=a_{1} \log D_{H}$, with the coefficient $a_{1} \ll 1$ for $W / t \geq 25$. (It is worth noting that a related quantity, normalized participation ratio [9], decays exponentially with the system size.) This implies wave function localization in the Hilbert space. The broad 
distribution of $S_{1}^{P}$ (Fig. 2d) indicates a large variance of dominant thermal bubble sizes in different eigenstates. We also calculated the rank-2 participation entropy and found its coefficient $a_{2} \ll 1\left(a_{2}=S_{2}^{P} / \log D_{H}\right)$, further verifying the localization of the system. It is worth mentioning that $a_{2} \neq a_{1}$ (the inset of Fig. 2(b)), indicating that this random interaction driven MBL phase is multifractal. The broad distribution of participation entropy $P\left(S_{1}^{p}\right)$ shown in Fig. 2 (e) is consistent with the multifractal behavior.

Fig. 2(c) shows the bipartite entanglement entropy. We find that it grows with increasing $L$ even for very strong random interactions (we have checked the entanglement scaling for $W / t$ up to $10^{6}$ ). At the same time, $S(L)$ apparently bends downwards for $W / t \geq 35$. We attribute the growth of $S(L)$ to finite size effect, as even at infinite randomness limit we still see strong $L$ dependence in $S(L)$ for $L$ up to 100 (Fig. 1(c)). In the distribution $P(s)$ shown in Fig. $2(\mathrm{f})$ we find $P(s \rightarrow 0)$ tends to diverge as $L$ increases. This signifies the robustness of insulating blocks for finite random interaction.

Entanglement dynamics and quantum nonergodicity. - To further verify the MBL phase, we study the quantum dynamics by initializing the system in random product states. The time-dependent entanglement entropy $(S(\tau))$ and number imbalance $(I(\tau))$ are monitored (Fig. 3). The number imbalance is defined as

$$
I(\tau)=\frac{N_{1}(\tau)-N_{0}(\tau)}{N_{1}(\tau)+N_{0}(\tau)},
$$

with $N_{1}\left(N_{0}\right)$ referring to number of particles in the initially occupied (unoccupied) lattice sites. For the number imbalance (Fig. 3(c)), we find that it does not relax at long time for large $W / t$, confirming the dynamical nonergodicity of the system. For $S(\tau)$ (Fig. 3(a)), we obtain a linear growth at the beginning up to a ballistic time scale $\tau_{0}$, and logarithmic growth at later time, which is qualitatively similar to the case of on-site disorder driven MBL. But there are two quantitative differences from the on-site disorder case. One is that the ballistic time scale $\tau_{0}$ is about several tunneling time even at huge $W$. We expect $\tau_{0}$ to be the tunneling time multiplied by the typical thermal-bubble size in our bubble-neck MBL phase. The other is that the long time limit of entanglement entropy $S(\infty)$ is significantly larger than the deep on-site disorder MBL phase, which we attribute to the existence of thermal bubbles in our MBL system.

The $M B L$ transition at finite $W / t$. - As we further decrease $W / t$, the cross-block couplings (Fig. 1 (a)) become more important and eventually drive a delocalization/thermalization transition. Fig. 4 shows the behavior of the different diagnostics. Fig. 4(a) shows the gap ratio $r$. At strong randomness $W / t>20, r$ approximately stays at the universal Poisson value. For $W / t<5$, we find that $r$ systematically approaches the GOE value $r_{G}$ with increasing $L$, which implies that the system is in a thermal phase. We expect $r(W / t)$ to approach a step
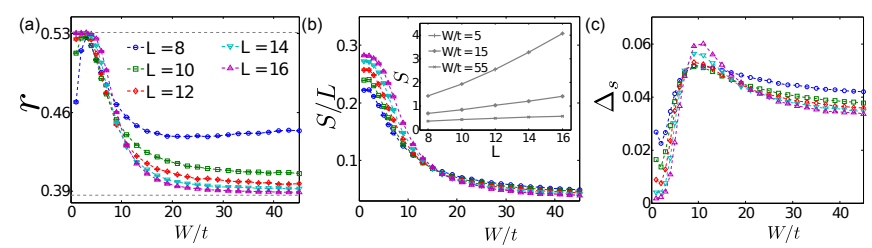

Figure 4. MBL transition of 1D fermions with random interactions. (a) Disorder averaged adjacent gap ratio $r$ as a function of the random-interaction strength $W / t$. The level statistics obey the GOE and Poisson distributions in the thermal and MBL phase with $r$ approaching 0.53 and $2 \log 2-1$ (marked by 'dashed' lines), respectively. The transition gets sharper as we increase the system size $L$. (b) Disorder averaged half-chain entanglement entropy density $S / L$. The inset shows the scaling of $S$ with increasing $L$. The entanglement entropy is strongly suppressed at large $W$. (c) Standard deviation of $s$ across disorder samples $\left(\Delta_{s}\right)$. The numeric results indicate a MBL transition locating at $W_{\mathrm{c}} / t \in(5,15)$.

function in the thermodynamic limit, giving a sharp transition at certain critical random-interaction strength $W_{c}$. The crossings for different lines in Fig. 4(a) indicate $W_{c} / t$ lies between 5 and 15. Fig. 4(b) shows the bipartite entanglement entropy density $(s=S / L)$. At small $W$, the entanglement entropy obeys volume-law scaling, and is expected to approach the thermal entropy $(\sim 0.35 L)$ for large enough $L$. We find that the entanglement entropy has a plateau-like behavior for small $W / t$, providing numerical evidence for $s$ to be a constant in the thermal phase. Fig. 4(c) shows the variance of entanglement entropy $\left(\Delta_{s}\right)$, which has been used to diagnose the MBL transition $[10,34,39,40]$. In calculating $\Delta_{s}$, we first average $s$ over all eigenstates within one disorder realization, and then calculate the standard deviation across different samples. In our study of the random interaction model, we see $\Delta_{s}$ developing a peak in the crossover regime. The peak value grows significantly as we increase $L$, which is qualitatively similar to what has been found for the random on-site disorder models [10, 34, 39]. This diverging behavior of the entanglement variance also suggests $W_{c} \in(5,15)$.

Conclusion. - We study random interaction driven MBL phase and point out its key differences with the on-site disorder driven case. We construct the generic bubble-neck eigenstates for the MBL phase in the infinite randomness limit, transcending the LIM description of MBL. With exact diagonalization, we confirm the MBL phase at finite random interaction by calculating level statistics, participation entropy and entanglement dynamics. At weak random interaction, we find that the system undergoes a thermalization transition which is cross-block-tunneling-driven. The random interaction driven MBL discussed in this paper is generic for onedimensional clean spinless fermions (as shown in [32] by studying different models) and is qualitatively different from MBL studied in interacting systems with singleparticle disorder. 
Acknowledgment. - This work is supported by JQINSF-PFC and LPS-MPO-CMTC. We acknowledge the University of Maryland supercomputing resources (http://www.it.umd.edu/hpcc) made available in conducting the research reported in this paper.

\section{Statistical Bubble Localization with Random Interactions-Supplementary Materials}

\section{S-1. STOCHASTIC SAMPLING OF THERMAL BUBBLES}

As shown in Fig. 1 in the main text, the eigenstates at infinite random interaction have a generic bubble-neck structure. In this section we discuss how to estimate the average entanglement entropy of such eigenstates with a stochastic sampling method.

Note that only the bubbles that cross the two links between sites $L / 2$ and $L / 2+1$, and between sites 1 and $L$ will contribute to the entanglement entropy (we use the periodic boundary condition). There are two different scenarios(i) a single bubble spreads over both links, and (ii) two disconnected bubbles with one over each link. The bubble configuration is then parametrized as

$$
\alpha=\left(z, l_{k \in[1, z]}, q_{k}, l_{k}^{\text {left }}\right),
$$

with $z=1,2$ representing the two different scenarios, $l_{k}$ and $q_{k}$ the size and particle number in each thermal bubble, $l_{k}^{\text {left }}$ the size of the thermal bubble within the left half of the system (with sites $j=1, \ldots L / 2$ ). Note that $\alpha$ can be thought as a function of either a Fock state or an eigenstate.

The thermal bubble involves two regions, the left (with sites restricted to $[1, L / 2]$ ) and the right (restricted to $[L / 2+1, L])$. The maximal entanglement entropy (EE) of this bubble configuration is

$$
S_{\max }(\alpha)=\sum_{k} \log m_{1}(\alpha, k),
$$

with $m_{1}(\alpha, k)$ the Hilbert space dimension of bubble-left-region or bubble-right-region depending on which one is smaller,

$$
m_{1}(\alpha, k)=\left[\sum_{\tilde{q}=0}^{q_{k}} D_{\text {therm }}\left(\min \left(l_{k}^{\text {left }}, l_{k}^{\text {right }}\right), \tilde{q}\right)\right],
$$

with $l_{k}^{\text {right }}=l_{k}-l_{k}^{\text {left }}$. Correspondingly we introduce $m_{2}(\alpha)=\sum_{\tilde{q}=0}^{q_{k}} D_{\text {therm }}\left(\max \left(l_{k}^{\text {left }}, l_{k}^{\text {right }}\right), \tilde{q}\right)$. Assuming the eigenstates in the thermal bubbles are approximately random states, the Page-value [33] estimate of EE $S_{\mathrm{pv}}(\alpha)$ for this bubble configuration is given by

$$
S_{\mathrm{pv}}(\alpha)=\sum_{k}\left(\sum_{p=m_{2}+1}^{m_{1} m_{2}} \frac{1}{p}-\frac{m_{2}-1}{2 m_{1}}\right) .
$$

Grouping the states with the same $\alpha$ together, the averaged EE (averaging over all eigenstates) can be rewritten as

$$
S_{\mathrm{avg}}=\sum_{\alpha}\left(\frac{D_{H}(\alpha)}{D_{H}}\right) S_{\max / \mathrm{pv}}(\alpha)
$$

with $D_{H}(\alpha)$ the number of states having the same $\alpha$. In numerics, the weight $D_{H}(\alpha) / D_{H}$ can be easily sampled by randomly sampling Fock states $|\{n\}\rangle$ (with equal weight) because the probability follows

$$
P\left[\alpha(|\{n\}\rangle)=\alpha_{0}\right]=D_{H}\left(\alpha_{0}\right) / D_{H} .
$$

\section{S-2. INTEGRABILITY OF THE THERMAL BUBBLE FOR THE NEAREST-NEIGHBOR-RANDOM-INTERACTION MODEL}

In this section, we show that the thermal bubble for the particular model in Eq.(1) is exactly solvable by mapping to "inflated fermions". For a given thermal-bubble many-body state, say $|1001001\rangle$, we can first add '0' in the front 
(the example state becomes $\| 01001001\rangle$, then the many-body state is made of ' 01 's and '0's. We can group '01' together and make it an inflated fermion denoted as $\mathbb{1} \equiv 01$. For the model in Eq.(1), we only have singleparticle tunnelings in the thermal bubble state. The tunneling Hamiltonian in the inflated fermion basis is completely identical to that of the original fermions. This can be proven by considering tunneling processes one by one, as the coupling from $|\ldots \mathbb{1} 0 \ldots\rangle$ to $|\ldots 0 \mathbb{1} \ldots\rangle$ in the inflated-fermion basis maps to the coupling between $|\ldots| \begin{array}{llll}0 & 1 & 0 & \ldots\end{array}$ and $|\ldots 001 \ldots\rangle$ in the original basis. This thermal bubble is then exactly solvable as the inflated fermions are non-interacting. The map also holds for hard core bosons.

Two remarks are in order. First, the inflated-fermion mapping restricts to models with homogenous tunnelings only. An inhomogeneous term like $h_{j} c_{j}^{\dagger} c_{j}$, induces a long-range string-like interaction between the inflated fermions, and the resulting model is no longer solvable. Second, the calculation of entanglement entropy using the inflated-fermion picture does not appear to be straightforward as the entanglement-cut may split one inflated fermion into two halves.

\section{S-3. THE THERMAL PHASE}
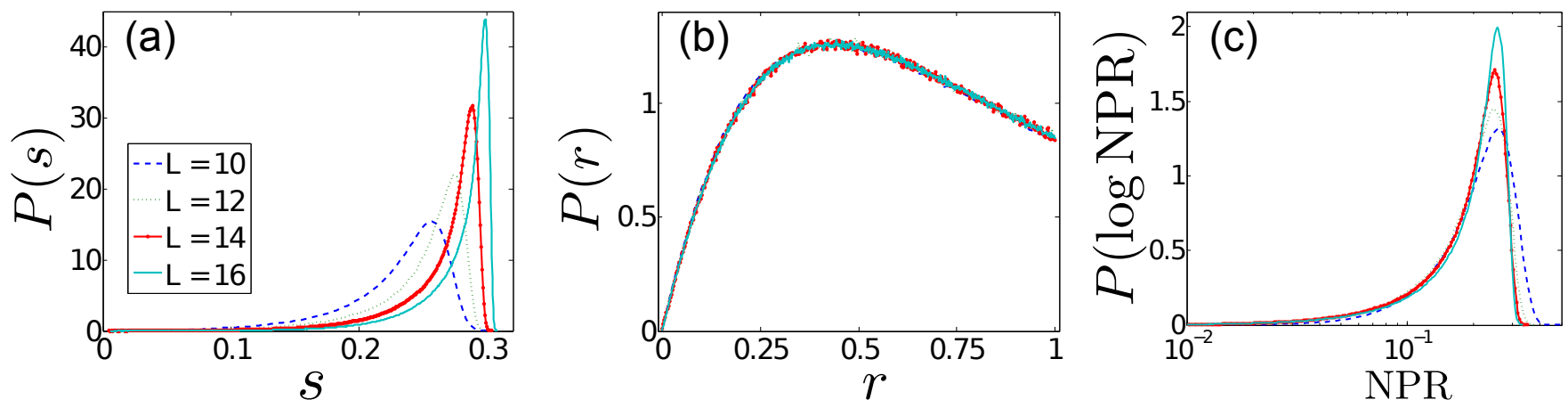

Figure S1. The quantum thermal phase in the random interaction model (Eq. (1) in the main text). (a), (b), and (c) show the probability distribution of half-chain entanglement entropy density $(P(s))$, the adjacent gap ratio $(P(r))$, and the normalized participation ratio $(P(\log \mathrm{NPR}))$, with a random interaction strength $W / t=3$. The thermal phase obeys the GOE level statistics as shown by $P(r)$ in (b). As we increase the system size $L$, the distributions $P(s)$ and $P(\operatorname{logNPR})$ get sharpen, implying the eigenstates are completely extended both in real space and in the Hilbert space at weak random interaction.

In this section, we give the results confirming thermalization of the random interaction model in the tunneling dominant regime. The probability distributions of the different diagnostics in the thermal phase are shown in Fig. S1. We see that the distributions for entanglement entropy and normalized participation ratio (NPR) develop sharp peaks at finite values of $s$ (entropy density) and $\log$ NPR, respectively (Fig. S1(a,b)). This implies that the thermal phase is completely extended both in real space and in the many-body Hilbert space. Furthermore, the probability distribution of $r$-value collapses to the GOE form even for small system sizes with deviations barely noticeable as shown in Fig. S1(b), providing strong numerical evidence for the many-body level repulsion in this model at weak random interaction. All in all, spinless fermions with random nearest neighbor interaction at weak randomness provide one ideal model to investigate quantum thermalization, despite the translationally invariant interacting case being integrable.

\section{S-4. CROSS-OVER FROM MBL TO THERMALIZATION}

In this section, we show the cross-over from MBL to thermalization. Fig. S2 shows how the probability distributions of entanglement entropy density and gap ration, $P(s)$ and $P(r)$, evolve in the crossover regime between thermal and localized phases. From Fig. S2(a), we see that $s$ has a very broad distribution in the crossover regime. For $W / t>15$, $P(s)$ has a strong peak at zero entanglement, indicating the dominance of insulating blocks.Upon decreasing $W / t$, the large-entanglement tail of $P(s)$ shifts rightward, corresponding to the increase in cross-block tunnelings. $P(r)$ is fairly robust at large $W / t$. As we decrease $W / t, P(r)$ quickly approaches the GOE distribution once it starts to deviate from the Poisson case. This strongly indicates GOE and Poisson distributions characterize two stable phases (thermal and MBL) in this model. 
(a)

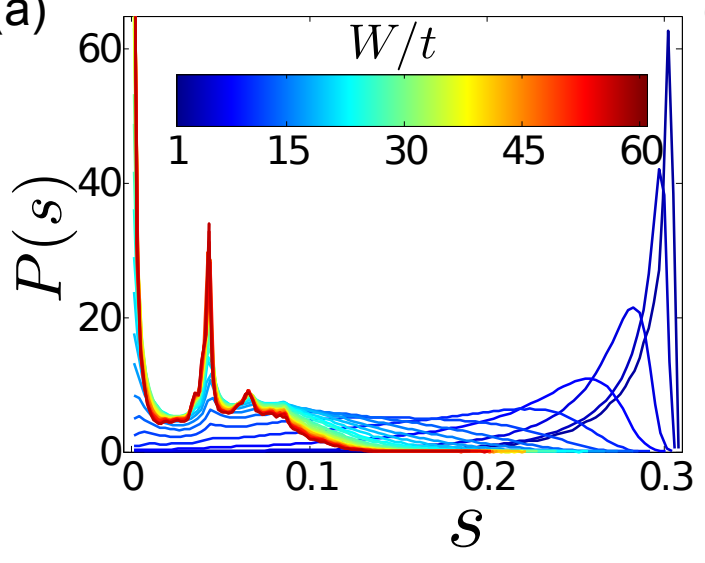

(b)

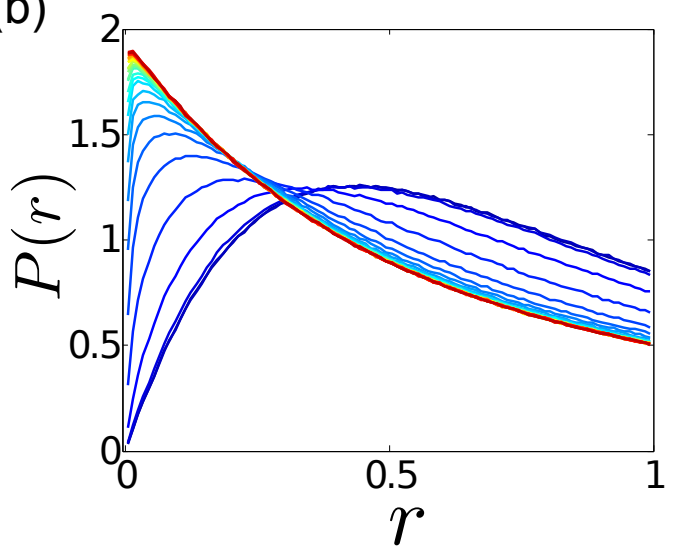

Figure S2. Cross-over from the thermal to MBL phases $(L=16)$. (a), and (b) show the distributions of the entanglement entropy density and gap ratio with varying $W / t$. In (b) we use the same color scheme to index $W / t$ as shown in (a).

\section{S-5. MBL IN THE INTERACTING AUBRY-ANDRÉ MODEL}

To show the MBL physics we present for random interactions is generic, we also provide the results for the AubryAndré (AA) model. On top of the original model (see Eq. (1) in the main text), we now add an incommensurate potential,

$$
\Delta H_{\mathrm{AA}}=2 \lambda \sum_{j} \cos (2 \pi Q j) c_{j}^{\dagger} c_{j}
$$

with $Q$ an irrational number (here we use golden ratio $Q=\frac{1+\sqrt{5}}{2}$ ). As shown in Fig. S3, we do not find any qualitative difference from the pure random interaction case if the incommensurate potential is weak with singleparticle Hamiltonian being extended. It is worth noting here that for the AA model the thermal bubble is no-longer integrable. We also mention that our numerical results (not shown) for the AA model in the localized single-particle case (i.e. $\lambda>t$ in contrast to Fig. S3 where $\lambda<t$ is considered explicitly) does not show any thermalization transition in the presence of random interactions implying that localized single-particle states remain localized when random interactions are turned on.
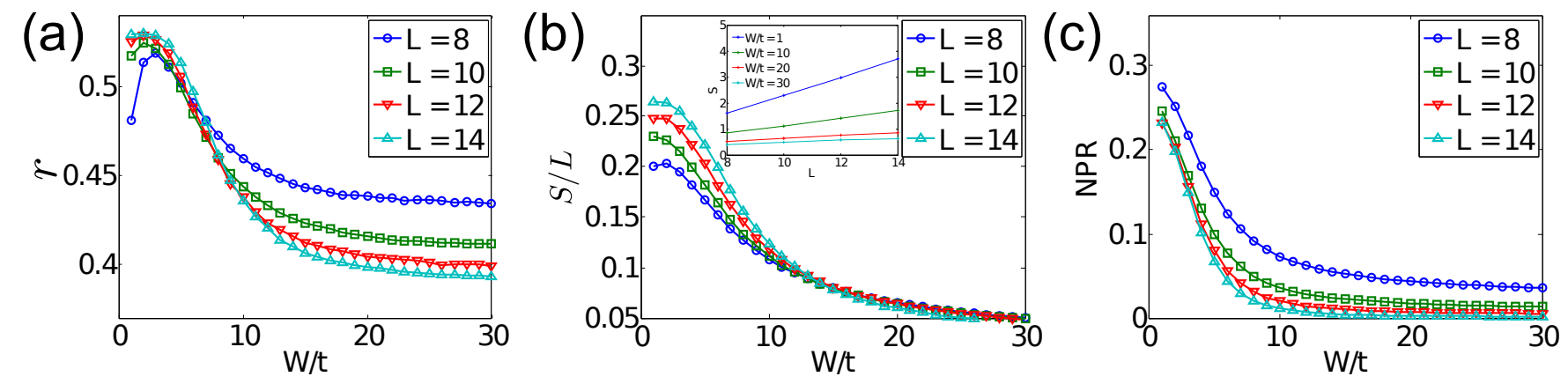

Figure S3. The MBL transition of the Aubry-André model with random interaction. (a) The averaged gap ratio. (b) The entanglement entropy. Its inset shows the entanglement entropy scaling with increasing system size. (c) The normalized participation ratio. Here we choose $\lambda / t=0.5$ where the single-particle AA Hamiltonian is extended. 


\section{S-6. MBL IN THE $(V+W) /(V-W)$ RANDOM INTERACTION MODEL}

As a second model, we modify the original model by adding a constant interaction

$$
\Delta H_{\mathrm{int}}=V \sum_{j} n_{j} n_{j+1} .
$$

Now the random interaction is drawn from $[V-W, V+W]$, instead of $[-W, W]$. As shown in Fig. S4, there is no qualitative difference from the the results presented in the main text.

(a)

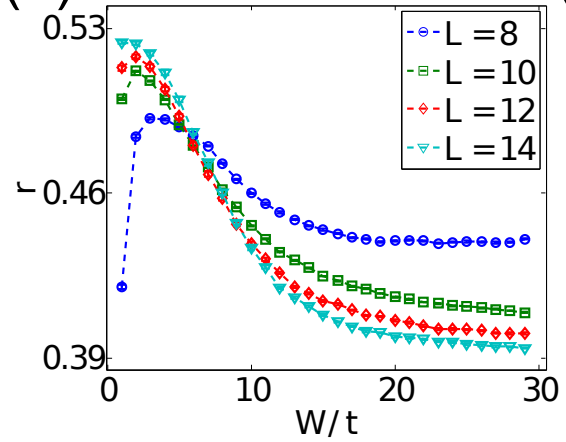

(b)

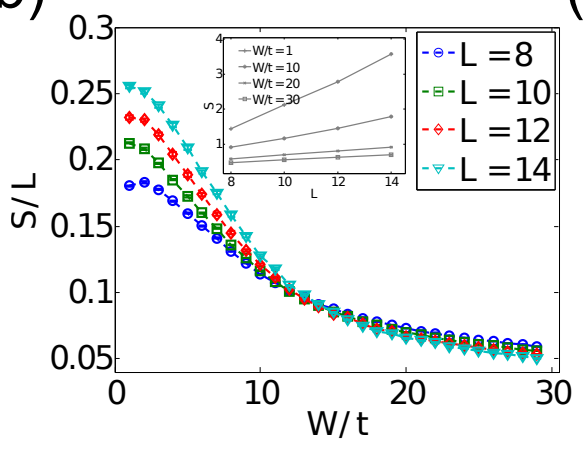

(c)

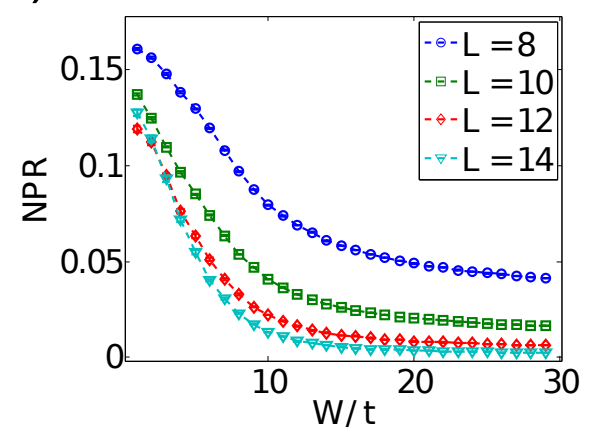

Figure S4. The MBL with random interaction with $V_{j} \in[V-W, V+W]$. (a) The averaged gap ratio. (b) The entanglement entropy. Its inset shows the entanglement entropy scaling with increasing system size. (c) The normalized participation ratio. Here we choose $V / t=4$.

[1] P. W. Anderson, Phys. Rev. 109, 1492 (1958).

[2] D. Basko, I. Aleiner, and B. Altshuler, Annals of Physics 321, 1126 (2006).

[3] R. Nandkishore and D. Huse, Annu. Rev. Conden. Matter Phys. 6, 15 (2015).

[4] E. Altman and R. Vosk, Annu. Rev. Condens. Matter Phys. 6, 383 (2015).

[5] V. Oganesyan and D. A. Huse, Phys. Rev. B 75, 155111 (2007).

[6] A. Pal and D. A. Huse, Phys. Rev. B 82, 174411 (2010).

[7] J. H. Bardarson, F. Pollmann, and J. E. Moore, Phys. Rev. Lett. 109, 017202 (2012).

[8] B. Bauer and C. Nayak, J. Stat. Mech. Theor. Exp. 2013, P09005 (2013).

[9] S. Iyer, V. Oganesyan, G. Refael, and D. A. Huse, Phys. Rev. B 87, 134202 (2013).

[10] J. A. Kjäll, J. H. Bardarson, and F. Pollmann, Phys. Rev. Lett. 113, 107204 (2014).

[11] T. Devakul and R. R. P. Singh, Phys. Rev. Lett. 115, 187201 (2015).

[12] V. Khemani, F. Pollmann, and S. L. Sondhi, Phys. Rev. Lett. 116, 247204 (2016).

[13] X. Yu, D. Pekker, and B. K. Clark, ArXiv e-prints (2015), arXiv:1509.01244 [cond-mat.str-el].

[14] S. P. Lim and D. N. Sheng, Phys. Rev. B 94, 045111 (2016).
[15] D. M. Kennes and C. Karrasch, Phys. Rev. B 93, 245129 (2016).

[16] J. Z. Imbrie, Journal of Statistical Physics 163, 998 (2016).

[17] M. Schreiber, S. S. Hodgman, P. Bordia, H. P. Lschen, M. H. Fischer, R. Vosk, E. Altman, U. Schneider, and I. Bloch, Science 349, 842 (2015), http://www.sciencemag.org/content/349/6250/842.full.pdf.

[18] P. Bordia, H. P. Lüschen, S. S. Hodgman, M. Schreiber, I. Bloch, and U. Schneider, Phys. Rev. Lett. 116, 140401 (2016).

[19] P. Bordia, H. Lüschen, U. Schneider, M. Knap, and I. Bloch, ArXiv e-prints (2016), arXiv:1607.07868 [condmat.quant-gas].

[20] J. yoon Choi1, S. Hild, J. Zeiher, P. Schau, A. RubioAbadal, T. Yefsah, V. Khemani, D. A. Huse, I. Bloch, and C. Gross1, Science 352, 1547 (2016).

[21] J. Smith, A. Lee, P. Richerme, B. Neyenhuis, P. W. Hess, P. Hauke, M. Heyl, D. A. Huse, and C. Monroe, Nat. Phys. (2016), 10.1038/nphys3783.

[22] D. A. Huse, R. Nandkishore, and V. Oganesyan, Phys. Rev. B 90, 174202 (2014).

[23] M. Serbyn, Z. Papić, and D. A. Abanin, Phys. Rev. Lett. 111, 127201 (2013).

[24] A. Chandran, I. H. Kim, G. Vidal, and D. A. Abanin, Phys. Rev. B 91, 085425 (2015). 
[25] V. Ros, M. Mller, and A. Scardicchio, Nuclear Physics B 891, 420 (2015).

[26] X. Li, S. Ganeshan, J. H. Pixley, and S. Das Sarma, Phys. Rev. Lett. 115, 186601 (2015).

[27] R. Modak and S. Mukerjee, Phys. Rev. Lett. 115, 230401 (2015).

[28] X. Li, J. H. Pixley, D.-L. Deng, S. Ganeshan, and S. Das Sarma, Phys. Rev. B 93, 184204 (2016).

[29] K. Hyatt, J. R. Garrison, A. C. Potter, and B. Bauer, ArXiv e-prints (2016), arXiv:1601.07184 [cond-mat.dis$\mathrm{nn}]$.

[30] R. Vasseur, A. J. Friedman, S. A. Parameswaran, and A. C. Potter, Phys. Rev. B 93, 134207 (2016).

[31] Y. Bar Lev, D. R. Reichman, and Y. Sagi, ArXiv e-prints (2016), arXiv:1607.04652 [cond-mat.dis-nn].

[32] See the Supplementary Material.
[33] D. N. Page, Phys. Rev. Lett. 71, 1291 (1993).

[34] V. Khemani, S. P. Lim, D. N. Sheng, and D. A. Huse, ArXiv e-prints (2016), arXiv:1607.05756 [cond-mat.dis$\mathrm{nn}]$.

[35] R. J. Bell, Reports on Progress in Physics 35, 1315 (1972).

[36] F. Wegner, Zeitschrift für Physik B Condensed Matter 36, 209 (1980).

[37] A. Rodriguez, L. J. Vasquez, K. Slevin, and R. A. Römer, Phys. Rev. B 84, 134209 (2011).

[38] D. J. Luitz, F. Alet, and N. Laflorencie, Phys. Rev. Lett. 112, 057203 (2014).

[39] D. J. Luitz, N. Laflorencie, and F. Alet, Phys. Rev. B 91, 081103 (2015).

[40] R. Vosk, D. A. Huse, and E. Altman, Phys. Rev. X 5, 031032 (2015) 